\title{
3D Modeling of 2D Computed Tomographic Projections
}

\author{
Padmapriya B ${ }^{\text {a,* }}$, Nandakumar Ravichandran ${ }^{\mathrm{a}}$

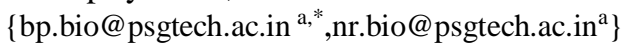 \\ a, ${ }^{*}$ Associate Professor, ${ }^{a}$ Department of Biomedical Engineering PSG College of Technology \\ Coimbatore, Tamilnadu, India
}

\begin{abstract}
D Computed Tomography is used to view and inspect the internal and external structures of an object in 3D space. It takes thousands of $2 \mathrm{D}$ projections with 360 degrees rotation of an object. They can be able to recreate objects with high resolution. It creates an illusion of depth in image. It became a very useful process in the field of medicine especially in imaging and analysis. .
\end{abstract}

Keywords: slices, region of interest, rendering, slices, modelling, computed tomography.

\section{Introduction}

2D image is a single slice of an image. Stack of 2D images is a 3D volume image. 3D CT allows us to study the anatomy by using software, which is available both as optional package and an auxiliary unit. One such software is 3D-DOCTOR which is an advanced 3D image rendering, processing, and analysis from image software. It is used to create 3D images by extracting information from image files. It can handle large volume of 3D images with efficient management implementation. 3D DOCTOR acts as an excel tool to process and analyse 3D images in an effective and efficient manner.

\section{Nomenclature}

\section{COMPUTED TOMOGRAPHY}

Computed Tomography is an advanced equipment used to diagnose and detect diseases, trauma, etc. It allows the user to take detailed 3D images of structures such as blood vessels, tissues, and bones. It is non-invasive and painless diagnostic tool. X-Ray attenuations by different tissues with the help of rows of detector and rotating X-Ray tube. The X-ray projections taken from different angles are processed and reconstructed as slices of an image, which gives the detailed information on the different tissues.

3D DOCTOR

3D-DOCTOR, approved by US Food and Drug Administration for medical imaging and 3D visualization applications, was developed by Able Software Corp. to provide a complete set of tools for visualizing 3D volume image data, including CT, MRI, Microscopy, and other types of imaging applications. Quantitative analysis can be performed using the tool and to other applications such as object measurement, etc. It supports both grayscale and color 
images. It supports image formats such as PNG, TIFF, DICOM, etc. It has efficient management implementation. The software is currently used by many leading hospitals, research organizations in the world.

\section{DISPLAY WINDOWS}

3D Doctor has five types of display windows - Image plane window, montage window, volume window, surface window, and plot window. A single slice of an image can be viewed in Image plane window whereas all slices of 3D images can be displayed in Montage window. Volume window displays volume rendered images. Surface window displays 3D surface models. Plot window enables the user to display histograms and measurements. The data displayed by the plot window can be saved in table format and used in spreadsheets for further purposes. Functions such as zoom in and out, contrast adjustment, etc. can be performed in Image plane window by clicking the view menu.

\section{OBJECT \& BOUNDARY}

Objects are defined by boundaries and the data of pixel content of the image lies within the boundaries. A 3D image can be split into multiple objects. Different 3D displays can be created by grouping objects. Boundaries define the area of $3 \mathrm{D}$ object of interest in the image. Boundaries give information to the user, which part of the image must undergo 3D rendering. The polarity rule is used to determine the included and excluded parts of the image.

\section{REGION OF INTEREST (ROI)}

A small region of interest can be drawn within the image and the drawn ROI can be reconstructed selectively. To produce a high resolution CT image with no artifacts, either there must be full field of view exposure or the ROI must be acquired in high dose and dose must be reduced outside the ROI. ROI can be defined in a medical image with tumour to measure its size.

\section{SEGMENTATION}

properties such as Gray level, contrast, etc. It allows the doctor or physician to closely examine the anatomical structure of a subject. One of the difficulties involved in segmentation is due to high variability in the medical images. The segmentation can be performed both manually and automated. Automatic segmentation can be done through the tool available in the software. Manually, segmentation can be performed by defining boundaries.

\section{INTERACTIVE SEGMENTATION}

Jia He, et al. ${ }^{1}$ in their book mentioned Interactive segmentation allows clear extraction of objects of interest, indicated by human interactions, based on high perception level. In other words, interactive segmentation can be used to separate an object from the background. The object of interest will be annotated by the user by defining boundaries. In recent studies, interactive segmentation is used to isolate tumour from healthy cells.

\section{SURFACE RENDERING}

Surface rendering allows us to create a 3D image of an object by acquiring dataset of the object. It collects the complete data of the object of interest, extracted by interactive segmentation. It can reconstruct images by taking only one part of the 3D dataset available. Recent study reveals that there is an extensive application of surface rendering in archaeology. 
Fragile objects are rendered through surface rendering for examination without causing any damage to it. One of the easiest methods to collect data of the object of interest and reconstructing image is by defining a threshold. High computational cost is the disadvantage of surface rendering.

\section{VOLUME RENDERING}

Volume rendering used to create 3D representation of data and it is not like surface rendering, which use surface representation. It displays both $3 \mathrm{D}$ images and its boundaries. It an create $2 \mathrm{D}$ projection from discretely sampled $3 \mathrm{D}$ data. It enables the doctors or patients to visualize the human anatomy better. This feature is used in extracting features of medical images taken by imaging devices Ultrasound, ECG, etc.

\section{Creation Of 3d Models - Pelvis Ct}

We can model 3D surface models from cross-section image using the tool. Initially, boundary lines must be generated for objects in the image and the model is created by surface rendering command. Multiple objects can be defined for each 3D images and status can be changed using Edit/ Object setting command. Then using boundary editor, you can generate boundaries after defining objects. Import and Export of boundary data from one program to another is applicable. The step-by-step process of creating a 3D model is explained as follows.

Click the File/Open command and open the 3D image as shown in Fig 1. For image with multiple slices, there will be two windows - montage window and image plane window. The montage window displays a montage of all the slides whereas the latter displays a single slice of image. If only one single slice of an image is displayed and montage window is not displayed, convert the 2D image files into a 3D image by using File $\rightarrow$ New stack as shown in Fig 2. The montage window and image plane window are shown in Fig 3. Avoid combining SI and other standard units, which can lead to confusion because the equations will be dimensionally unbalanced. If it is compulsory to use mixed units, specify the quantities with the units in the equation[3].

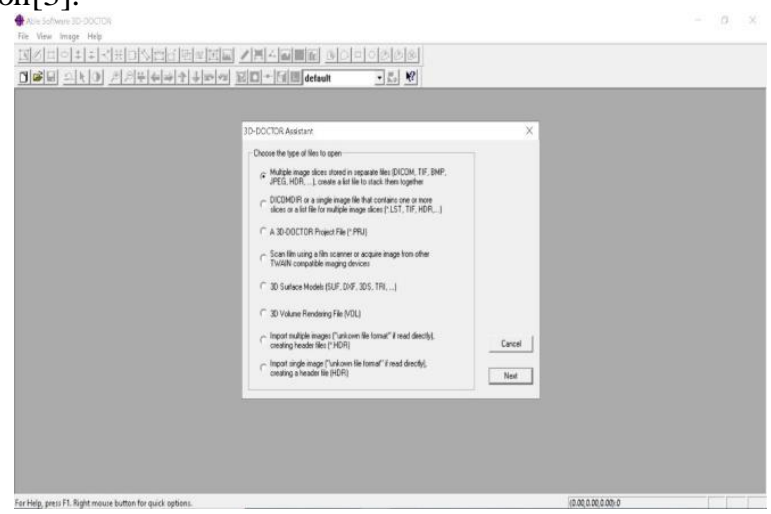

Fig 1. Opening the 3D image 


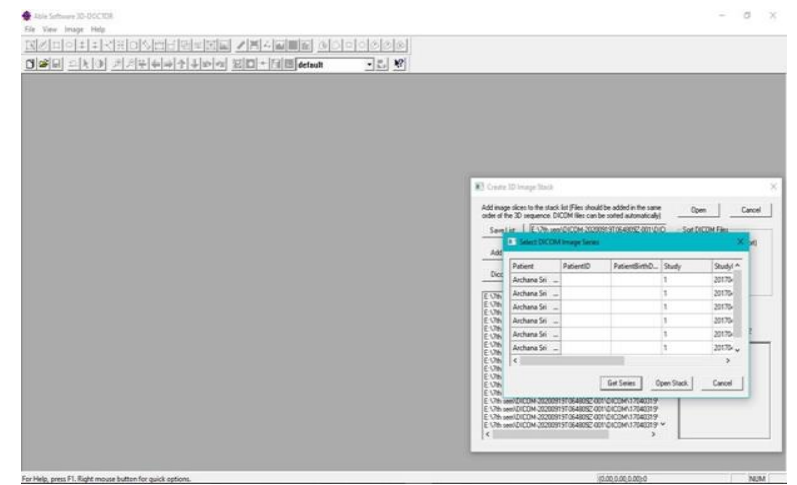

Fig 2. Converting 2D image into 3D image

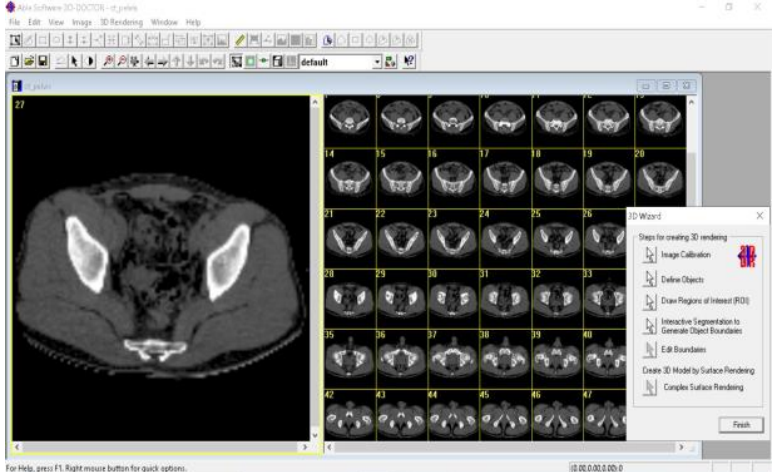

Fig 3. Montage window and Image plane wiindow

For automatic detection of object boundaries, select the 3D Rendering/Auto Segment command and enter the number of how many possible objects that is to be detected. The image that we segmented is shown in Fig 4. After detection of boundaries, if the results are reliable, we can directly execute the Step-6, or else we can continue with the consecutive steps [1].

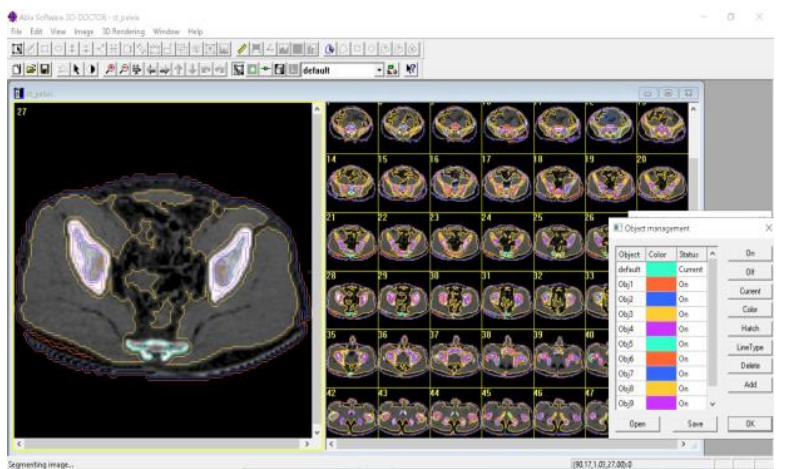

Fig 4. Automatic detection of object boundaries

Add new object names for the image by using Edit/Object Settings command. We can create new objects by creating a new object name and make them as current object by clicking the 'Add' button. As a result, boundary data will be generated from the segmentation process as shown in Fig 5. 


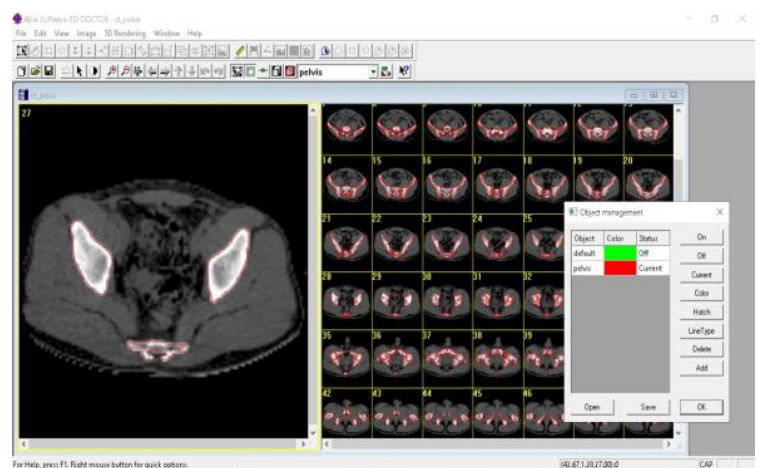

Fig 5. Creating new object names

We can limit the segmentation area by defining the Region of Interest using Edit/Region of Interest command. Boundary extraction is performed by considering the pixels lie within the ROI. Polygons can be drawn within the image after turning on the ROI. Shapes such as circle, square, etc. are available to draw the desirable ROI. ROIs are displayed as blue thick lines as shown in Fig 6.

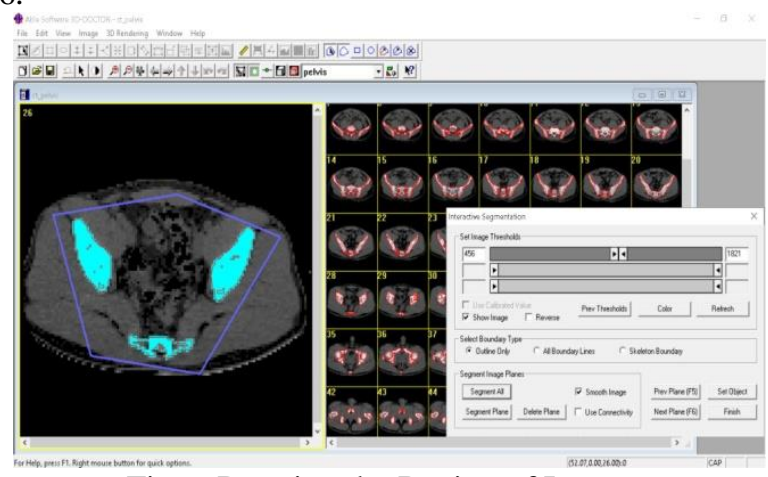

Fig 6. Drawing the Region of Interest

\section{Results}

The results of the experiment are explained below.

Select the 3D Rendering/Interactive Segmentation command and keep the current object for segmentation. The Image plane window display would have been changed as shown in Fig 7. The red colour indicates pixels of threshold having values between maximum and minimum values. We can adjust the slide bar to change minimum and maximum values. 


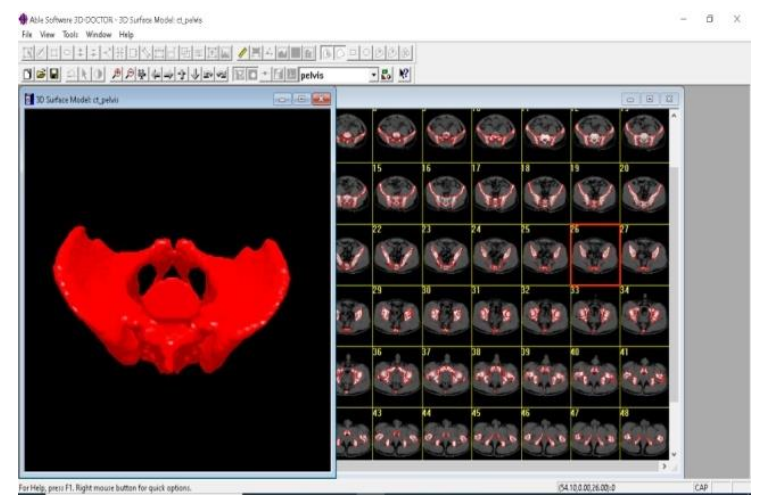

Fig 7. Interactive segmentation

- Repeat the same procedure and boundaries can be segmented using the tools in the Edit/Boundary Editor. Using 3D rendering/ Split Object command, objects can be split into two sub-objects. Save the boundaries and other data using File/Save/Save Project command.

- $\quad$ Then, 3D rendering $\rightarrow$ Simple Surface Rendering or the 3D rendering $\rightarrow$ Complex Surface commands, we can design 3D surface models as shown in Fig 8.

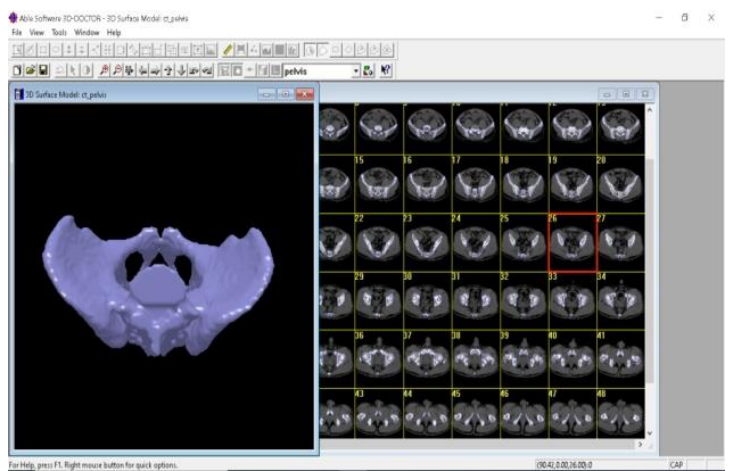

Fig 8. Complex Surface Rendering

- $\quad 3 \mathrm{D}$ volume rendering can be performed 3D Rendering $\rightarrow$ Volume Rendering command as shown in Fig 9.

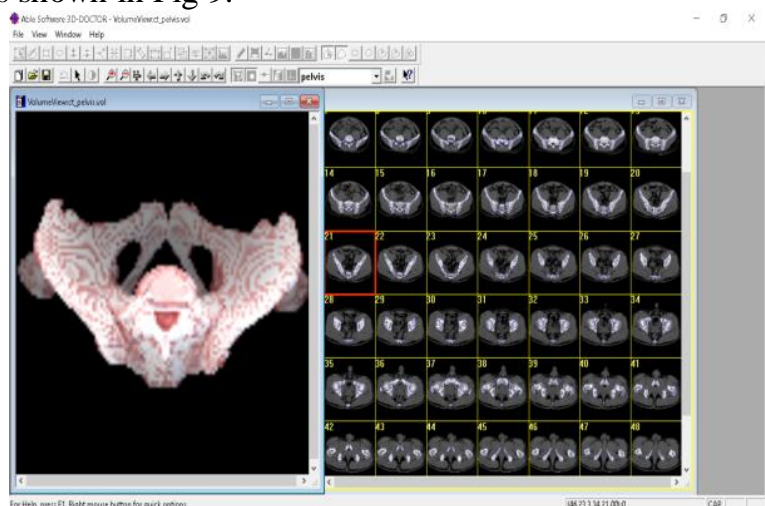

Fig 9. Volume Rendering 
- Save the file in stl format. Using a standard stl file viewer, import the segmented stl file and view the 3D model in the required orientation as shown in Fig 10, $11 \& 12$. Attributes such as display, colour, and orientation can be changed.

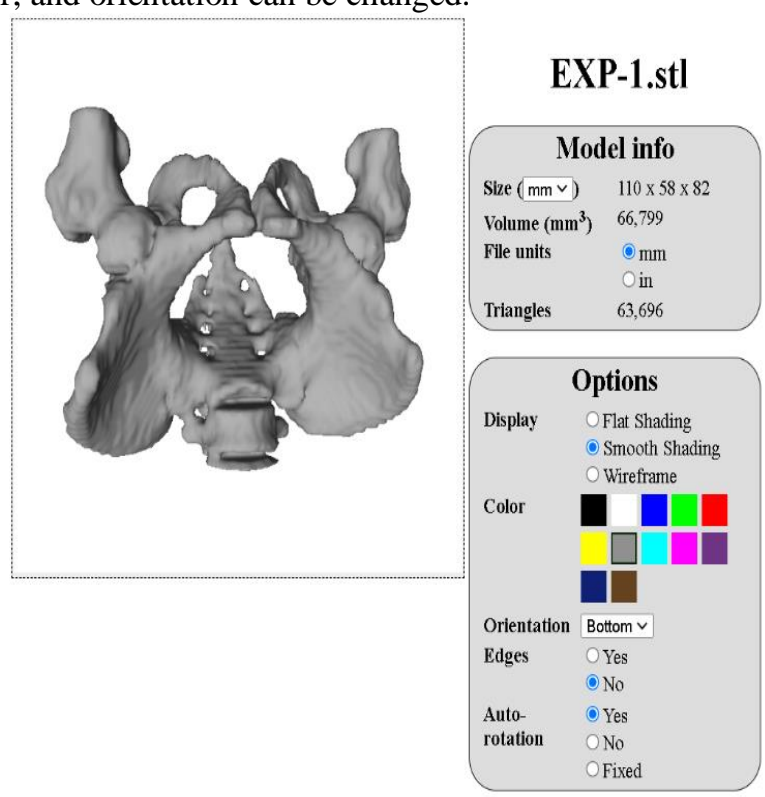

Fig 11. Bottom view of the 3D model

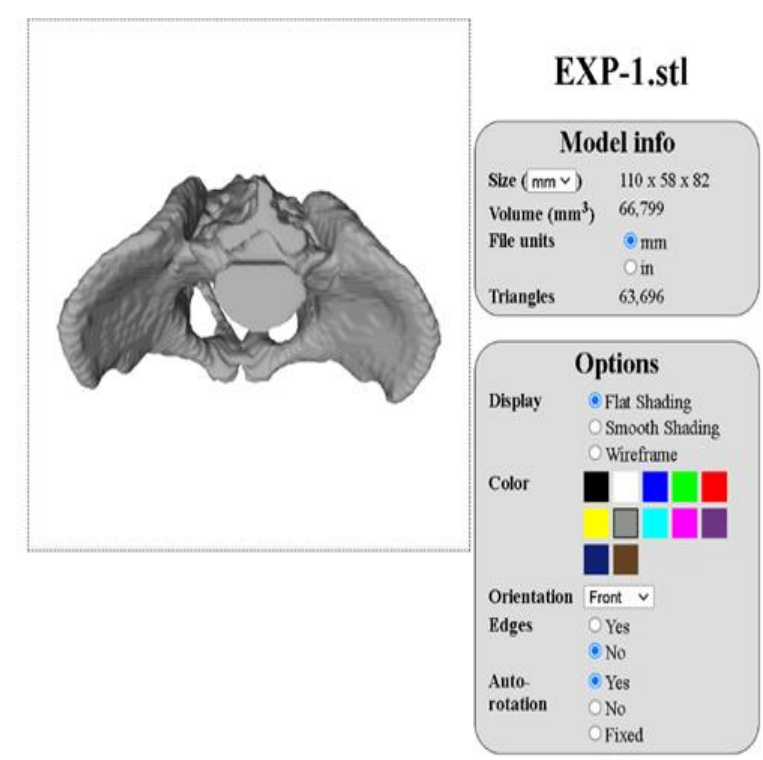

Fig 12. Frontal view of the 3D model 


\section{Creation Of 3d Models - Skull Ct}

The results yielded when the same experiment carried out using CT images of skull are as follows [2].

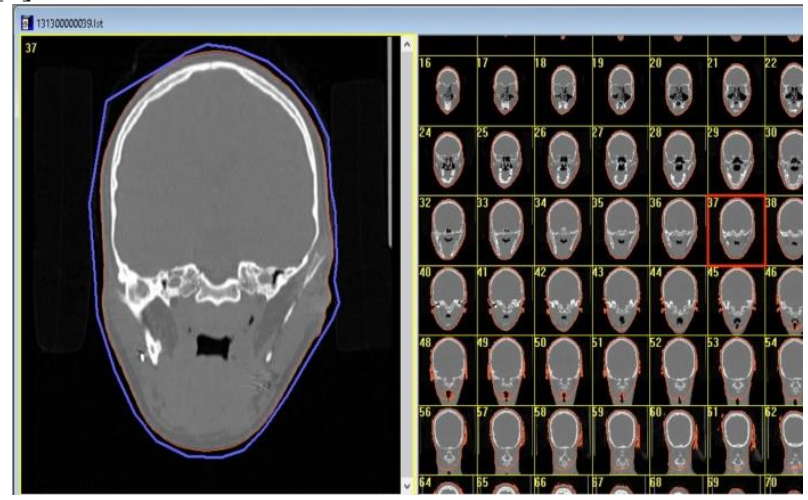

Fig 13. Defining the ROI

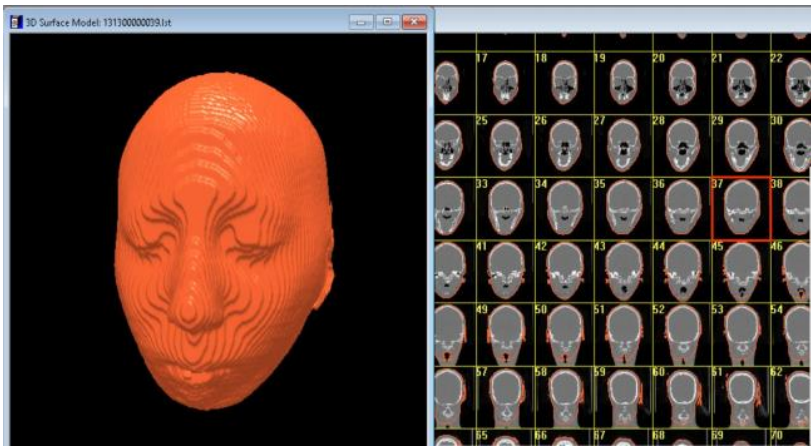

Fig 14. Complex Surface Rendering

\section{Conclusion}

3D CT used in 3D printing, academia, research, engineering, and earth sciences. Recently it has become a useful factor to assist in quality control process. 3D imaging is interactive and helps in gathering information on movements, controls, etc. Recently, research has been in this field to understand the physiological process of organs in animals and humans. 


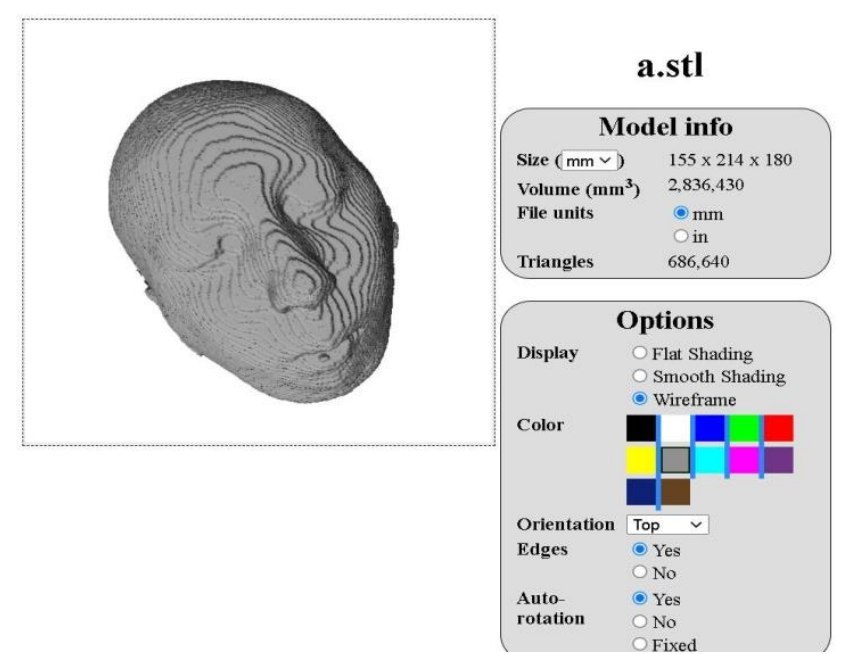

Fig 16. Online STL viewer

\section{References}

[1] Jia He, Chang-Su Kim, C. - C. Jay Kuo. "Interactive Segmentation Techniques" Algorithms and Performance Evaluation, Springer Nature, 2014.

[2] Y. Ted Wu, "From CT Image to 3D Model", Advanced Imaging, Aug. 2001, pp. 20-23.

[3] Vernon, Tim \& Peckham, Daniel, "The benefits of 3D modeling and animation in medical teaching", The Journal of audiovisual media in medicine, 25. 